\title{
Endoscopic management of splenic pseudocysts associated with acute and chronic pancreatitis
}

\author{
Surinder Singh Rana ${ }^{a}$, Ravi Sharmaa, Puneet Chhabra, ${ }^{a, c}$, Vishal Sharmaa, Rajesh Guptab, \\ Deepak Kumar Bhasina,c
}

Post Graduate Institute of Medical Education and Research (PGIMER), Chandigarh; Fortis Hospital, Mohali, Punjab, India

\begin{abstract}
Background Splenic pseudocysts (SP) are a rare consequence of both acute and chronic pancreatitis. Surgery has been conventional treatment for SP and literature on role of endoscopic treatment is scant. In this study, we retrospectively evaluated SP clinical and radiological characteristics as well as the outcome following endoscopic drainage.

Methods Retrospective analysis of SP patients seen at our unit from January 2002 to June 2015. All patients were treated with attempted endoscopic transpapillary drainage with a nasopancreatic drain or stent. Patients not responding underwent endoscopic ultrasound-guided transmural or percutaneous radiological drainage.

Results Eleven patients with SP (all male; mean age: $40.5 \pm 8.8$ years) were studied. Seven patients had chronic pancreatitis and 4 patients had SP following acute pancreatitis. The majority (10/11; $91 \%$ ) had alcohol-related acute or chronic pancreatitis with one patient having coexistent pancreas divisum. Seven (64\%) patients were treated successfully with transpapillary drainage only; one (9\%) patient needed combined transpapillary and transmural drainage; and 3 (27\%) patients needed surgery.
\end{abstract}

Conclusion Endoscopic transpapillary drainage is an effective treatment for SP especially when it is not infected and with clear contents, and is associated with partial ductal disruption that can be bridged by an endoprosthesis.

Keywords Pancreas divisum, chronic pancreatitis, acute pancreatitis, stent, nasopancreatic drain, endotherapy, pancreatic stricture, endoscopic ultrasound, pseudocyst, spleen

Ann Gastroenterol 2016; 29 (3): 1-5

\section{Introduction}

Pancreatic pseudocysts are usually located in peri-pancreatic area but can occasionally occur at atypical locations like liver, spleen, mediastinum, pelvis, and kidney [1-7]. Pseudocysts in

Departments of a Gastroenterology (Surinder Singh Rana, Ravi Sharma, Puneet Chhabra, Vishal Sharma, Deepak Kumar Bhasin); 'burgery (Rajesh Gupta), Post Graduate Institute of Medical Education and Research (PGIMER), Chandigarh; ' $\mathrm{G}$ astroenterology and Hepatology, Fortis Hospital, Mohali, Punjab (Puneet Chhabra, Deepak Kumar Bhasin), India

Conflict of Interest: None

Correspondence to: Prof. Deepak K. Bhasin, House No. 1041, Sector 24B, Chandigarh, 160023 India, Tel.: +91 172 2725056, Fax: +91 172 2744401, e-mail: deepakkbhasin@gmail.com

Received 24 January 2016; accepted 30 March 2016; published online 25 April 2016

DOI: http://dx.doi.org/10.20524/aog.2016.0038 these atypical locations are rare and pose difficult diagnostic and therapeutic challenge. Splenic pseudocysts (SP) are rare and have been previously reported as case reports with most of them being treated with surgery [7]. Literature supporting endoscopic drainage of SP is scant [1-7].

In this study, we describe clinical and radiological characteristics of SP in 11 patients ( 4 of whom had been earlier published as case reports or their data had been included in a paper published earlier) $[4,5,7]$ as well as our experience with endoscopic drainage and clinical outcome in these patients.

\section{Patients and methods}

We performed a retrospective analysis of patients with SP seen at our unit from January 2002 to June 2015. Clinical records were reviewed to identify patient symptoms and imaging findings. Patients were referred to us for endoscopic drainage and were treated by attempted endoscopic 
transpapillary drainage. All patients were symptomatic, and had SP documented on contrast-enhanced computed tomography (CECT) scan. Endoscopic ultrasound (EUS) has been available at our institution for the past 6 years and patients seen during that time frame also underwent EUS examination (EG-3670 URK radial echoendoscope or EG-3870 UTK linear echoendoscope Pentax Inc., Tokyo, Japan; GF-UE160-AL5 radial echoendoscope or GF-UCT 180 linear echoendoscope; Olympus Pvt. Ltd., Gurgaon, India). All patients provided informed consent prior to endoscopic treatment and protocol was approved by institutional ethics committee.

Patients were treated with attempted endoscopic transpapillary drainage with a nasopancreatic drain (NPD) or pancreatic stent. Intravenous ciprofloxacin or ofloxacin was administered for antibiotic prophylaxis and hyoscine butyl bromide was used to inhibit duodenal peristalsis. Endoscopic retrograde pancreatography (ERP) was performed by standard technique using a TJF 145 or TJF 160 or TJF-Q180V (Olympus Pvt. Ltd., Gurgaon, India) side-viewing duodenoscope under conscious sedation using intravenous midazolam. Pancreatic duct (PD) disruption was defined by free extravasation of contrast outside the ductal system after contrast injection of main PD or dorsal duct (in patients with pancreatic divisum). PD disruption was defined as complete when main duct upstream to disruption was not visualized and as partial when the main duct was visualized upstream from site of disruption. After confirming disruption, a 5/7Fr stent or 5-Fr NPD was placed across papilla into the PD. An attempt was made to place endoprosthesis across disruption.

Following ERP, patients with NPD placement, were instructed to empty drainage bag and record daily drain output. They were advised to report back if there was no drainage for $24 \mathrm{~h}$ or color of the output changed to bilious, indicating displacement of NPD into duodenum. When blockage was suspected (no output for $24 \mathrm{~h}$ ), NPD was flushed with sterile saline and flow established by suction using a disposable syringe. The patients with stent placement were asked to report back if there was worsening of abdominal pain or onset of new symptoms. All these patients were followed up every 2 weeks for: 1) clinical evaluation, and 2) abdominal ultrasound. CECT of abdomen was done when there was complete clinical recovery along with complete resolution of pseudocysts on abdominal ultrasound.

The patients who did not respond to transpapillary drainage or had worsening of abdominal pain or had new onset/persistent fever with leukocytosis underwent repeat imaging. Patients with the same or increased size of pseudocyst underwent percutaneous radiological drainage or EUS-guided transmural single time complete aspiration with $19 \mathrm{G}$ needle or surgery after an interdisciplinary consultation involving a gastroenterologist and a surgeon.

Therapeutic success was defined as symptomatic improvement with radiological resolution of all pseudocysts on CECT of abdomen and therapeutic failure was defined as need for surgical intervention at any time. Following resolution, the stent/NPD was removed and a repeat pancreatogram obtained to document healing of ductal disruption. Thereafter, these patients were regularly followed up in our clinic.

\section{Results}

Eleven patients with SP (all male; mean age \pm SD: $40.5 \pm 8.8$ years; age range: $28-60$ years) were studied (Table 1 ). Seven patients had chronic pancreatitis and 4 patients had acute pancreatitis. Patients with acute pancreatitis presented 8 to 32 weeks after the onset of acute attack. All patients had abdominal pain and 2/11 (18\%) patients had also fever. Five (45\%) patients complained of left upper quadrant discomfort/ heaviness. The size of SP ranged from 2.5 to $15 \mathrm{~cm}$ (median $6 \mathrm{~cm})$.

SP were well demonstrated on CECT (Fig. 1). Magnetic resonance cholangiopancreatography (MRCP) was done in 3 patients (Fig. 2) and ductal communication was noted in $1(33 \%)$ patient. EUS was performed in 5 patients with good

Table 1 Profile of patients with splenic pseudocysts

\begin{tabular}{|c|c|c|c|c|c|c|c|c|c|}
\hline $\begin{array}{l}\text { Age } \\
\text { (yrs) }\end{array}$ & Sex & Etiology & $\begin{array}{l}\text { Pleural } \\
\text { effusion }\end{array}$ & $\begin{array}{l}\text { Size } \\
(\mathrm{cm})\end{array}$ & Disruption & Stent/NPD & $\begin{array}{l}\text { Period of } \\
\text { resolution }\end{array}$ & $\begin{array}{l}\text { EUS } \\
\text { drainage }\end{array}$ & Surgery \\
\hline 32 & $\mathrm{M}$ & Idiopathic & Yes & 9 & Single & 5 Fr stent & 2 weeks & Yes & No \\
\hline 44 & M & Alcohol & No & 15 & Single & 5 Fr NPD & 8 weeks & No & No \\
\hline 35 & $\mathrm{M}$ & Alcohol+PD & No & 8 & Single & 5 Fr NPD & 6 weeks & No & No \\
\hline 60 & M & Alcohol & Yes & 2.5 & Single & 5 Fr NPD & 8 weeks & No & No \\
\hline 42 & M & Alcohol & Yes & 10 & Single & 5 Fr stent & - & Yes & Yes \\
\hline 46 & M & Alcohol & Yes & 6 & Single & 7 Fr stent & 6 weeks & No & No \\
\hline 36 & M & Alcohol & No & 4 & Single & 5 Fr NPD & 5 weeks & No & No \\
\hline 42 & M & Alcohol AP & No & 3 & Single & $5 \mathrm{Fr}$ stent & 6 weeks & No & No \\
\hline 28 & M & Alcohol AP & No & 4 & Single & 5 Fr stent & 5 weeks & No & No \\
\hline 34 & $\mathrm{M}$ & Alcohol AP & Yes & 4 & Complete & 5 Fr stent & - & No & Yes \\
\hline 46 & M & Alcohol AP & Yes & 6 & Single & 5 Fr stent & - & No & Yes \\
\hline
\end{tabular}

AP, acute pancreatitis; $M$, male; $P D$, pancreas divisum; $N P D$, nasopancreatic drain; EUS, endoscopic ultrasound 


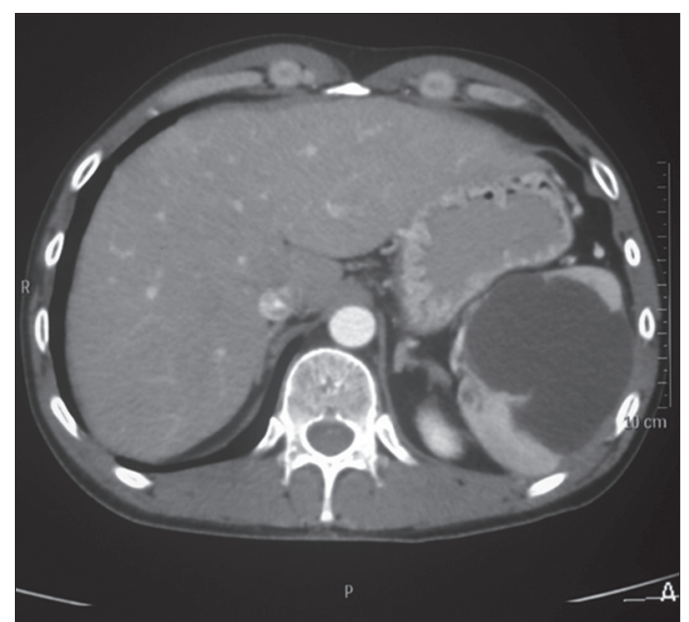

Figure 1 Computed tomography showing a large splenic pseudocyst

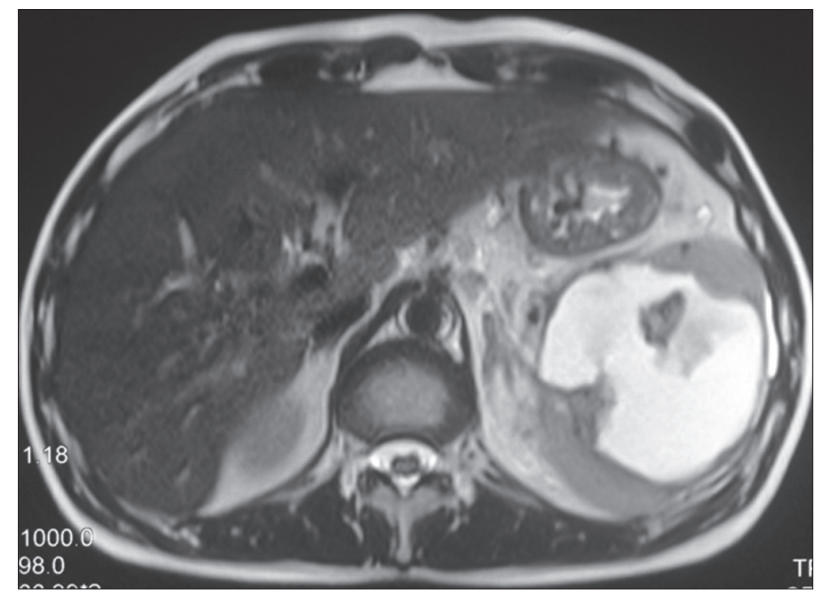

Figure 2 Magnetic resonance imaging of the abdomen showing a large splenic pseudocyst

visualization of SP and echogenic contents were noted in 3 patients.

All 11 patients underwent attempted endoscopic transpapillary drainage through the major $(\mathrm{n}=10)$ or minor papilla $(n=1)$. Ten patients had single partial disruption of main PD at tail region of pancreas (Fig. 3) whereas one patient with acute pancreatitis had complete disruption at neck region. A bridging endoprosthesis was placed in all 10 patients with partial disruption (Fig. 4) whereas non bridging stent was placed in patient with complete disruption. A $5 \mathrm{Fr}$ stent was placed in 6 patients, $5 \mathrm{Fr}$ NPD in 4 patients, and $7 \mathrm{Fr}$ stent in 1 patient, respectively. None had stricture or calculus in PD.

\section{Outcome in patients with chronic pancreatitis $(n=7)$}

Five (71\%) patients had marked improvement in symptoms following transpapillary drainage. In these patients, resolution of SP as well as associated other pseudocysts and pleural effusion was observed within 8 weeks (median 6 weeks). Another 2 patients who had fever, echogenic contents on

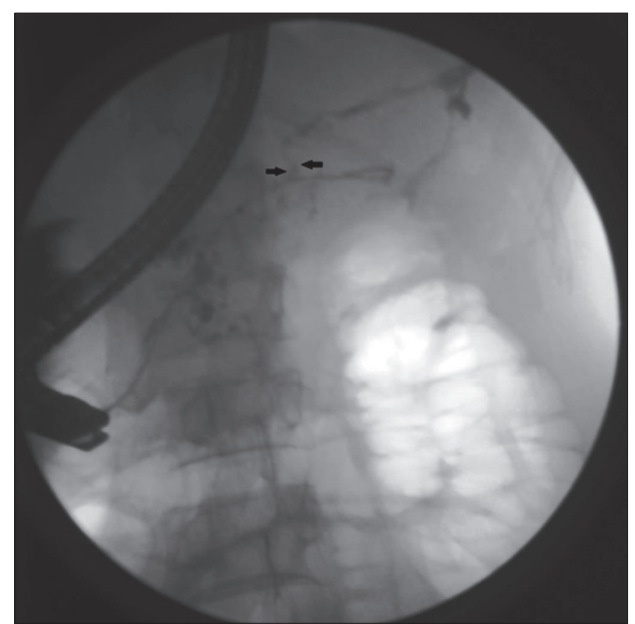

Figure 3 Endoscopic retrograde cholangiopancreatography showing a partial disruption at tail end. Guide wire negotiated across disruption

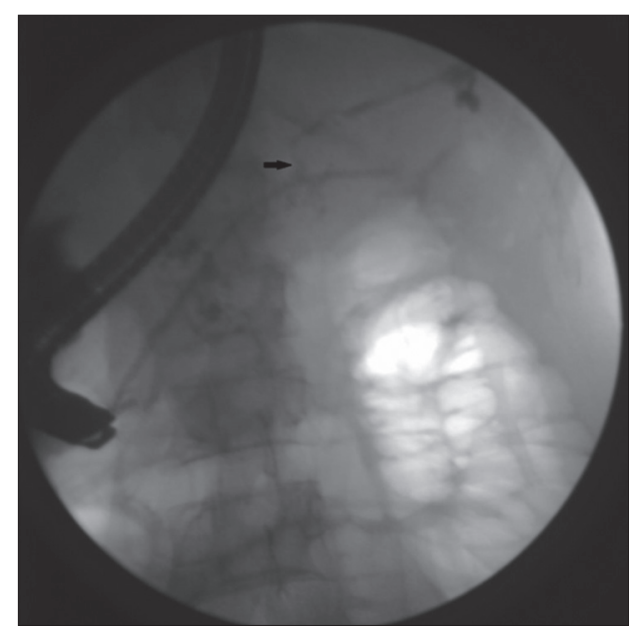

Figure 4 Endoscopic retrograde cholangiopancreatography showing bridging transpapillary stent placed across disruption

EUS and large size of pseudocyst ( 9 and $10 \mathrm{~cm}$ respectively) continued to be febrile with no relief in pain or decrease in size of pseudocyst. Therefore, these two patients underwent single time EUS-guided complete aspiration of the splenic pseudocyst 48 and $96 \mathrm{~h}$ of transpapillary drainage (Figs. 5, 6). Following aspiration of purulent material, both these patients had improvement and became afebrile within $48 \mathrm{~h}$. One of these patients had uneventful recovery and was discharged. The other patient had massive gastrointestinal bleed on the $7^{\text {th }}$ day of endoscopic transmural drainage and computed tomographic (CT) angiography revealed a $2.3 \mathrm{~cm}$ splenic artery pseudoaneurysm. As patient had hemodynamic compromise, emergency surgery was performed and patient had an uneventful post operative course.

On follow up, one patient with large SP $(15 \mathrm{~cm})$ developed chronic gastric volvulus after healing, possibly because of perigastric adhesions. There has been no recurrence of pseudocysts in these successfully treated patients over a follow up period of 14 months to 13 years. 


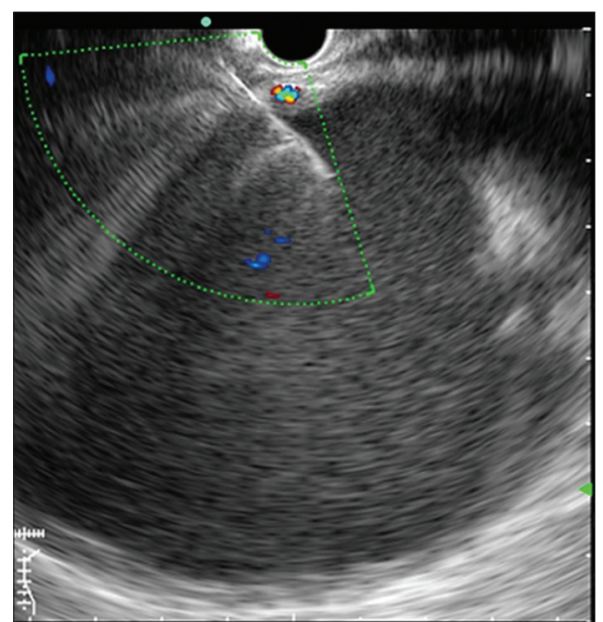

Figure 5 Endoscopic ultrasound-guided transmural aspiration of splenic pseudocyst. Echogenic contents can be noted

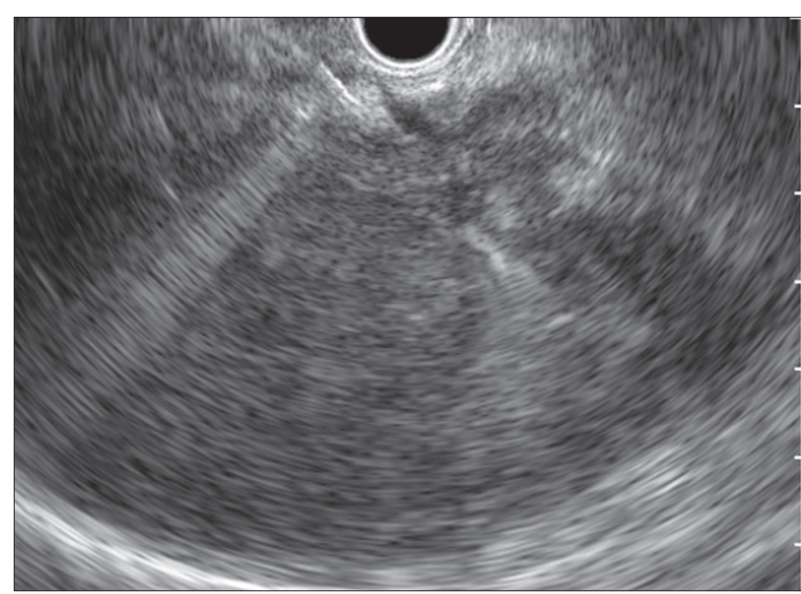

Figure 6 The splenic pseudocyst has been completely emptied

\section{Outcome in patients with acute pancreatitis $(n=4)$}

Two patients had partial PD disruption and they had clinical improvement following endoscopic transpapillary drainage. Resolution of pseudocysts including SP as well as pleural effusion was observed within 6 weeks and there has been no recurrence over a follow up of 8 months and 2 years respectively.

The patient with complete duct disruption continued to have abdominal pain and follow up imaging revealed increase in the size of pseudocyst. A single time complete aspiration of splenic pseudocyst was done under EUS guidance. Following this, patient remained pain-free for one week and thereafter patient had recurrence of pain along with fever. CT revealed recurrence of SP with ascites and patient was treated by surgery.

The other patient had gastrointestinal bleeding on the $12^{\text {th }}$ day following transpapillary drainage and CT angiography revealed a $2.1 \mathrm{~cm}$ splenic artery pseudoaneurysm. Urgent angiography was performed and pseudoaneurysm was embolized using coils. Following this patient had severe abdominal pain and CT revealed ascites with splenic necrosis and therefore patient underwent surgery.

\section{Discussion}

In spite of close proximity of the spleen to the pancreatic tail, SP are rare complication of both acute as well as chronic pancreatitis with majority of them occurring in adult patients with alcohol-induced pancreatitis [1-10]. A large series of 500 patients with chronic pancreatitis, reported only $5(1 \%)$ patients with SP [8]. Similarly, another study of 238 patients with pseudocysts, reported splenic involvement in 5.9\% patients only [9]. SP have been very rarely reported in acute pancreatitis [11]. A study of 100 patients with acute pancreatitis who were studied with CECT reported no patient with SP [12]. The SP result either from dissection of the splenic parenchyma by pseudocyst or tracking of pancreatic fluid into the spleen along the course of splenic vessels [10].

SP usually do not have any specific signs and symptoms with majority of patients presenting with abdominal pain. Presence of left upper quadrant heaviness or left pleural effusion may suggest splenic involvement as was seen in $45 \%$ and $55 \%$ of our patients $[8-10,13]$. Because of lack of specific symptoms and signs, CECT and MRCP play an important role in diagnosis and follow up of patients. MRCP can also delineate ductal anatomy and also occasionally demonstrate communication of SP with PD. EUS is also a useful investigational modality for evaluating pseudocysts because by determining solid necrotic debris it helps in planning optimal therapy [8].

Because of rarity, ideal management of SP is not clear and surgery in the form of splenectomy with or without distal pancreatectomy, cyst resection or drainage has been commonly used as therapeutic modality [5]. There are few reports of successful treatment by percutaneous radiological drainage but risk of external pancreatic fistula especially in patients with PD abnormality limits its use $[5,9,15]$.

While endoscopic transmural and/or transpapillary drainage has been successfully used in patients with abdominal pseudocysts, there is paucity of endoscopic data in patients with SP $[2,4,5,7]$. We have previously published case reports of successful resolution of SP with endoscopic transpapillary drainage using NPD or stent or combination of EUS-guided transmural aspiration with transpapillary drainage $[2,4,5,7]$. In this study also, 8/11 (73\%) patients with SP who underwent endoscopic intervention had resolution of all the pseudocysts within 8 weeks.

Of eleven patients, 10 patients had partial disruption which could be bridged in all and $8(80 \%)$ patients had successful outcome. It has been earlier demonstrated that patients with partial disruption respond better to endoscopic treatment than those with complete disruption [5]. In our study, 3 patients with echogenic contents in SP on EUS did not respond to transpapillary drainage alone. One of these could be successfully treated with combined transpapillary and transmural drainage, whereas other two required surgery. This observation is in accordance with previously published reports 


\section{Summary Box}

\section{What is already known:}

- Pancreatic pseudocysts are usually located in peripancreatic area

- Occasionally occur at atypical locations like liver, spleen, mediastinum, pelvis, and kidney

- Splenic pseudocysts are rare and are usually treated with surgery

\section{What the new findings are:}

- Endoscopic transpapillary drainage is a safe and effective modality for treatment of splenic pseudocysts especially when they are not infected, there is partial ductal disruption, there is no solid necrotic debris, and the disruption can be bridged by an endoprosthesis

that have shown that endoscopic transpapillary drainage alone has poor results in fluid collections with solid debris $[5,14]$.

In conclusion, SP is rare complication of both acute as well as chronic pancreatitis and endoscopic transpapillary drainage is safe and effective modality for its treatment especially when it is non-infected with clear contents and is associated with partial ductal disruption that can be bridged by an endoprosthesis.

\section{References}

1. Lehman GA. Pseudocysts. Gastrointest Endosc 1999;49:S81-S84.

2. Bhasin DK, Rana SS, Chandail V, et al. Intrahepatic pancreatic pseudocyst successfully treated by endoscopic transpapillary nasopancreatic drainage alone. JOP (online) 2005;6:593-597.

3. Bhasin DK, Rana SS, Chandail VS, et al. Successful resolution of a mediastinal pseudocyst and pancreatic pleural effusion by endoscopic nasopancreatic drainage. JOP (online) 2005;6:359-364.

4. Bhasin DK, Udawat HP, Rana SS, et al. Intra-splenic pancreatic abscess successfully treated by endoscopic transpapillary drainage through the minor papilla. Gastrointest Endosc 2005;62:192-194.

5. Bhasin DK, Rana SS, Nanda M, et al. Endoscopic management of pancreatic pseudocysts at atypical locations. Surg Endosc 2010;24:1085-1091.

6. Bhasin DK, Rana SS, Rao C, et al. Clinical presentation, radiological features, and endoscopic management of mediastinal pseudocysts: experience of a decade. Gastrointest Endosc 2012;76:1056-1060.

7. Rana SS, Chaudhary V, Sharma V, Sharma R, Dutta U, Bhasin DK. Infected pancreatic pseudocyst of spleen successfully treated by combined endoscopic transpapillary stent placement and transmural aspiration. Gastrointest Endosc 2014;79:360-361.

8. Malka D, Hammel P, Lévy P, et al. Splenic complications in chronic pancreatitis: prevalence and risk factors in a medical-surgical series of 500 patients. Br J Surg 1998;85:1645-1649.

9. Heider R, Behrns KE. Pancreatic pseudocysts complicated by splenic parenchymal involvement: results of operative and percutaneous management. Pancreas 2001;23:20-25.

10. Patil PV, Khalil A, Thaha MA. Splenic parenchymal complications in pancreatitis. JOP 2011;12:287-291.

11. Hastings OM, Jain KM, Khademi M, Lazaro EJ. Intrasplenic pancreatic pseudocyst complicating severe acute pancreatitis. Am J Gastroenterol 1978;69:182-186.

12. Mortelé KJ, Mergo PJ, Taylor HM, Ernst MD, Ros PR. Splenic and perisplenic involvement in acute pancreatitis: determination of prevalence and morphologic helical CT features. J Comput Assist Tomogr 2001;25:50-54.

13. Lankisch PG. The spleen in inflammatory pancreatic disease. Gastroenterology 1990;98:509-516.

14. Rana SS, Bhasin DK, Sharma RK, Kathiresan J, Gupta R. Do the morphological features of walled off pancreatic necrosis on endoscopic ultrasound determine the outcome of endoscopic transmural drainage? Endosc Ultrasound 2014;3:118-122.

15. Tennoe B, Bay D, Rosales R. Percutaneous drainage of intrasplenic pancreatic pseudocyst: case report. Acta Radiol 1996;37:195-197. 